\title{
CORRIGENDUM
}

\section{Patent ductus arteriosus treatment in preterm infants - time to consider shunt volume?}

PJ McNamara and A Jain

Journal of Perinatology (2013) 33, 497; doi:10.1038/jp.2013.48

Correction to: Journal of Perinatology (2013) 33, 248-249; doi:10.1038/jp.2012.104

Following publication of this letter, the authors noted that there were errors in the list of affiliations. The correct affiliations are shown below.

PJ McNamara ${ }^{1,2,3}$ and A Jain Ji,3 $^{2,4}$

${ }^{1}$ Department of Pediatrics, Hospital for Sick Children, Toronto, Ontario, Canada
${ }^{2}$ Department of Physiology \& Experimental Medicine Program, Hospital for Sick Children, Toronto, Ontario, Canada

${ }^{3}$ Department of Physiology, University of Toronto, Toronto, Ontario, Canada

${ }^{4}$ Department of Pediatrics, Mount Sinai Hospital, Toronto, Ontario, Canada 